\title{
Connectivity in Selfish, Cooperative Networks
}

\author{
Erwu Liu, Qinqing Zhang, and Kin K. Leung
}

\begin{abstract}
This paper studies the connectivity of large cooperative ad hoc networks. Unlike existing work where all nodes are assumed to transmit cooperatively, the cooperative network we consider is realistic as we assume that not all nodes are willing to collaborate when relaying other nodes' traffic. For such selfish, cooperative network, we use stochastic geometry and percolation theory to analyze the connectivity and provide an upper bound of critical node density when the network percolates.
\end{abstract}

Index Terms-Selfish node, cooperative network, connectivity.

\section{INTRODUCTION}

W HILE connectivity [1] is arguably the most critical performance metric for a wireless network to function well, one can not assume that any two nodes keep connected at all times due to fading, mobility, etc. Among various approaches for connectivity in wireless networks, cooperative communication shows great potential. The rationale for the extraordinary interest in exploiting cooperative techniques in wireless networks is very clear, as demonstrated by the high volume of publications in recent years [1]-[4], showing concrete advantages and potentials of cooperation. The challenges of achieving cooperation are not just technical but indeed multi-disciplinary, involving for instance understanding individual and social behavioral patterns and their impact on the performance of a wireless network. An important point is that, in addition to the purely technical cooperation, user's decision on joining such a network and its manner to cooperate are integral parts of the whole cooperative process [5], and thus new aspects related to user selfishness will come into the scene. Putting these together, it is of great interest to investigate the connectivity in cooperative networks with selfish nodes. Unfortunately, for cooperative networks, the connectivity problem and selfish behavior are separately considered [1], [5]-[7].

Our objective is to quantify the connectivity for cooperative ad hoc networks with user selfishness. As a first-step, this letter asymptotically studies the connectivity problem of cooperative networks with $p$-Selfishness (a simple model for selfish behavior, detailed in Section II), and provides an upper bound of critical node density for such systems to percolate.

\section{SySTEM Model}

Ad hoc nodes are randomly located and full connectivity of the whole network is generally not possible. Practically,

Manuscript received July 5, 2010. The associate editor coordinating the review of this letter and approving it for publication was F. Granelli.

E. Liu and K. K. Leung are with the Department of Electrical and Electronic Engineering, Imperial College, London, UK (e-mail: \{erwu.liu, kkleung\}@ieee.org).

Q. Zhang is with the Department of Computer Science, Johns Hopkins University, USA (e-mail: qinqing.zhang@jhuapl.edu).

Digital Object Identifier 10.1109/LCOMM.2010.091010.101173

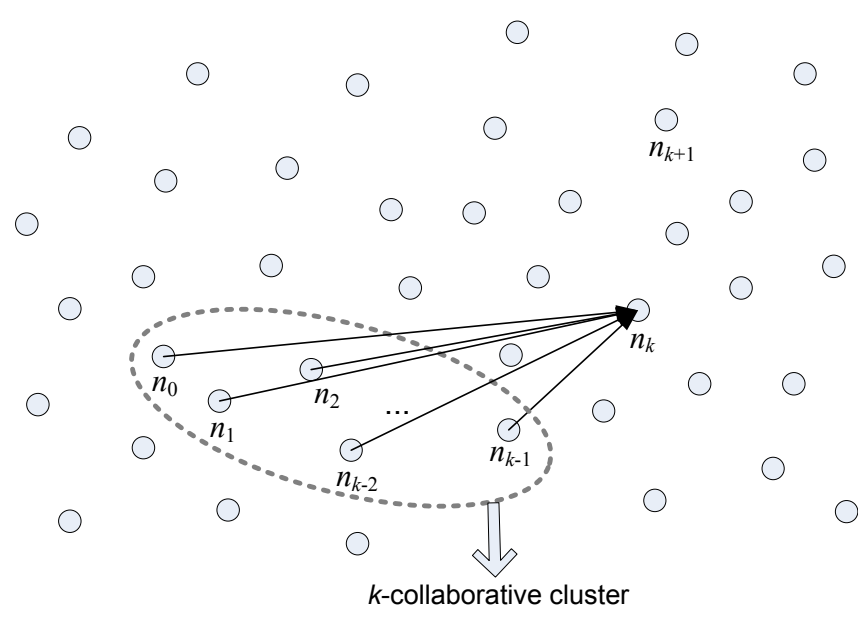

Fig. 1. Cooperative networks: $k$-collaborative cluster.

it is often sufficient for the network operator to ensure that some fraction of nodes instead of all nodes in the network are connected, whose occurrence in infinite large networks is mathematically defined as percolation [8]. By Kolmogorov's zero-one law, a network of infinite size will percolate once the node density $\lambda$ or node range $r$ is above some threshold. The density (or range) threshold for percolation to occur is called the critical density (or range), denoted by $\lambda_{c}\left(\right.$ or $r_{c}$ ).

Now consider a cooperative ad hoc network shown in Fig. 1. We assume that $k$ nodes $n_{0} \sim n_{k-1}$ transmit to Node $n_{k}$ in a cooperative manner. These $k$ nodes form a $k$-collaborative cluster. Because of cooperative transmission, the distance between $n_{k}$ and $n_{i}(i=0 \sim k-1)$ could be greater than node range $r$. Node $n_{k}$ and $k-1$ nodes out of $n_{0} \sim n_{k-1}$ together form another $k$-collaborative cluster and will reach Node $n_{k+1}$. In most works on cooperative transmission, it is assumed that all nodes in a cluster are willing to cooperatively transmit at all time. This is not true especially when nodes have some intelligence or social characteristic such as self-awareness. For example, nodes may become selfish in relaying other nodes' traffic and would not cooperatively transmit at some time. Our task is to analyze the $k$-collaborative connectivity problem for a cooperative network with selfish behavior. To be specific, we want to answer the question how many nodes are needed in each cluster for percolation to occur in a cooperative network with selfishness.

For achievability for cooperative ad hoc networks, we use the distributed frequency-shift keyed (FSK) cooperation method [1], which does not require phase coherence at the transmitters and represents a worst case of cooperation that achieves only power summing. Refer to Fig. 1, nodes are distributed across an infinite region according to a Poisson Point Process (PPP) with node density $\lambda>0$. $k$-collaborative connectivity for such network is defined as the existence of 


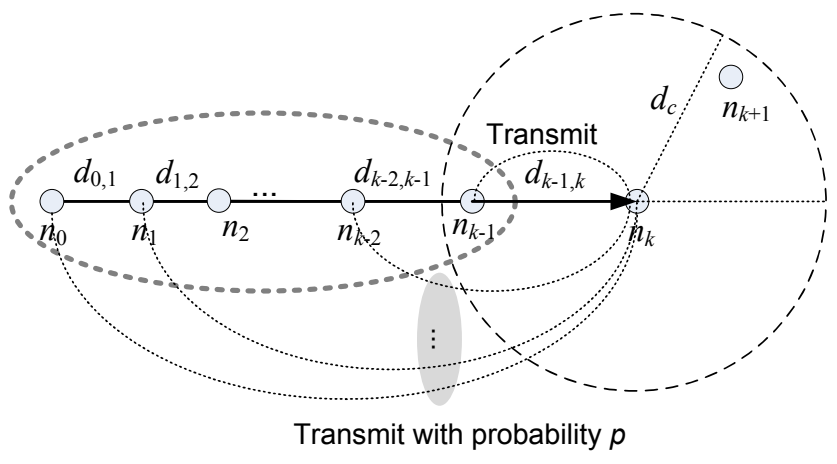

Fig. 2. $\quad k$-collaborative cluster with $p$-selfishness: worst-case cooperation.

one cluster chain containing an infinite number of connected clusters, each of which has $k$ nodes cooperatively transmitting in a selfish manner.

We assume that each node transmits with power $P_{t}$ and denote the received power at reference distance $d_{0}$ as $P_{0}$. Let $r$ be the node range, $P_{r}$ be the minimum receiving power required for successful receive. Using the modified Friis propagation model [9], we have $P_{r}=P_{0} \times\left(\frac{r}{d_{0}}\right)^{-\alpha}$, where $\alpha$ is the path loss exponent ${ }^{1}$. For any Node $k$ to successfully receive, the receiving power $P_{k}$ should be greater than $P_{r}$. We use $d_{i, j}$ to denote the distance between two nodes $n_{i}$ and $n_{j}$, and $P_{i, j}$ the power at Node $n_{j}$ received from Node $n_{i}$. Refer to Fig. 1, with node cooperation, the received power at Node $n_{k}$ depends on the power received from each branch and also on the selfish pattern of nodes. As a first step, this letter considers a simple model to describe selfish behavior. With this model, a node in a $k$-collaborative cluster will cooperatively transmit with a fixed probability $p$ when relaying other nodes' traffic. This model is likewise used in [6] to study cooperation in ad hoc networks, and we call it $p$-Selfishness throughout the letter. With $p$-Selfishness, the extreme case of $p=0$ corresponds to the non-cooperative scenario and $p=1$ the traditional cooperative scenario, respectively. Without loss of generality, we assume that Node $n_{k-1}$ has data for Node $n_{k}$ and it transmits to $n_{k}$ with probability 1 while all other nodes in the cluster cooperate with $n_{k-1}$ and transmit to $n_{k}$ with probability $p$. Since we are using the distributed FSK scheme [1] for node cooperation, the received power $P_{k}$ at Node $n_{k}$ is simply the power sum of all branches,

$$
\begin{aligned}
P_{k} & =P_{k-1, k}+\sum_{i=0}^{k-2} P_{i, k} \\
& =P_{0} \times\left(\frac{d_{k-1, k}}{d_{0}}\right)^{-\alpha}+\sum_{i=0}^{k-2} p \times P_{0} \times\left(\frac{d_{i, k}}{d_{0}}\right)^{-\alpha} .
\end{aligned}
$$

Now consider the worst case of cooperative transmission in a cluster. This corresponds to the one that produces the minimum power at $n_{k}$. Refer to Fig. $2, \forall i=0 \sim k-2$, with triangle inequality, we have $d_{i, k} \leq \sum_{i=0}^{k-2} d_{i, i+1}+d_{k-1, k}$. So

\footnotetext{
${ }^{1}$ The path loss exponent $\alpha$ is experimentally determined, and is typically in the range of 2 to 5 depending on propagation environment. For example, $\alpha=2.0$ is for free space, $2.5 \sim 3.0$ for rural areas, $3.0 \sim 4.0$ for urban areas, and $4.0 \sim 5.0$ for dense urban areas.
}

for a cluster of any topology, the received power $P_{k}$ satisfies

$$
\begin{aligned}
P_{k} \geq P_{0} & \times\left(\frac{d_{k-1, k}}{d_{0}}\right)^{-\alpha} \\
& +\sum_{i=0}^{k-2} p \times P_{0} \times\left(\frac{\sum_{m=i}^{k-1} d_{m, m+1}}{d_{0}}\right)^{-\alpha}=P_{k}^{L} .
\end{aligned}
$$

where $P_{k}^{L}$ is the power at Node $n_{k}$ received from the linear topology cluster shown in Fig. 2.

Define a positive $d_{c}$ so that $d_{c} \geq \max _{i=0, \cdots, k-1} d_{i, i+1}$. We have,

$$
\begin{aligned}
P_{k}^{L} & \geq P_{0} \times\left(\frac{d_{c}}{d_{0}}\right)^{-\alpha}+\sum_{i=0}^{k-2} p \times P_{0} \times\left(\frac{\sum_{m=i}^{k-1} d_{c}}{d_{0}}\right)^{-\alpha} \\
& =P_{0} \times\left(\frac{d_{c}}{d_{0}}\right)^{-\alpha} \times\left(1+\sum_{i=0}^{k-2} p \times(i+2)^{-\alpha}\right) .
\end{aligned}
$$

Obviously, if $P_{r}=P_{0} \times\left(\frac{d_{c}}{d_{0}}\right)^{-\alpha} \times\left(1+\sum_{i=0}^{k-2} p \times(i+2)^{-\alpha}\right)$, we will have $P_{k} \geq P_{k}^{L} \geq P_{r}$, i.e., the $k$-collaborative cluster with $p$-Selfishness can cooperatively transmit to Node $n_{k}$ successfully. We thus have

$$
d_{c}=r \times\left(1+\sum_{i=0}^{k-2} p \times(i+2)^{-\alpha}\right)^{\frac{1}{\alpha}} .
$$

According to [8], we have the following definition,

Definition 1 the critical node density $\lambda_{c}$ is given by

$$
\lambda_{c}=\inf \{\lambda: \theta(\lambda)>0\}
$$

where $\theta(\lambda)$ is the probability that an infinitely large path exists, given node density $\lambda$.

Equivalently, Definition 1 tells that if an infinitely large path exists with non-zero probability, then the network percolates.

Next we state two existing lemmas before presenting our result.

From percolation theory, we have the following important lemma on connectivity [10]

Lemma 1 (Scale Property) A 2-dimensional network with a range $r$ and a node density $\lambda$ has the same connectivity properties as a 2-dimensional network with a range $a \cdot r$ $(a>0)$ and $a$ node density $\frac{\lambda}{a^{2}}$

On the other hand, based on stochastic geometry [11], [10] provides the following lemma.

Lemma 2 (PPP Distance) If nodes are distributed according to a PPP with node density $\lambda$, the probability that the distance $d$ between a node and its nearest neighbor is less than $R$ is

$$
\operatorname{Prob}\{d \leq R\}=1-e^{-\pi R^{2} \lambda}
$$

Using Lemma 1 and Lemma 2, together with Definition 1, we then obtain an upper bound of node density for the considered network to percolate, 


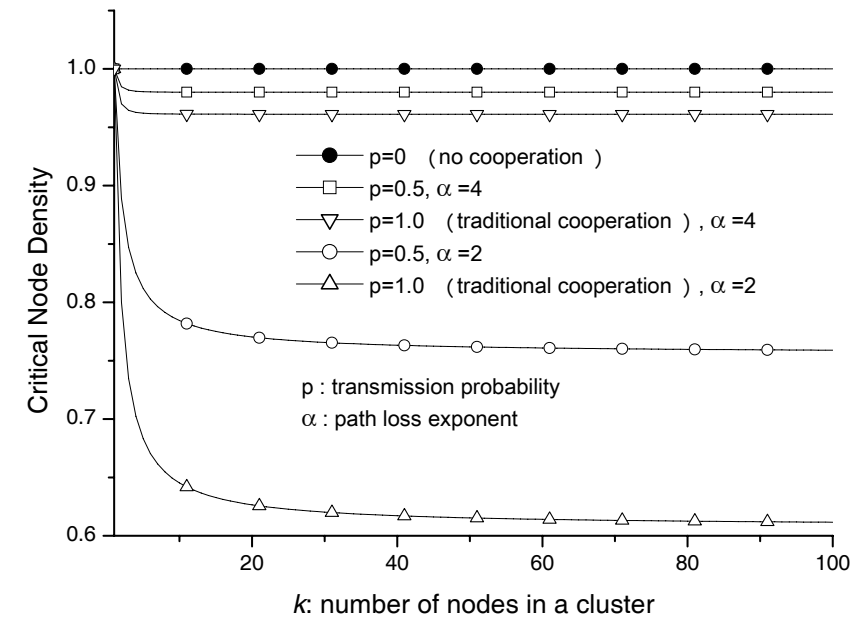

Fig. 3. Critical density in cooperative networks with $p$-selfishness.

Theorem 1 For a k-collaborative network with p-Selfishness, the critical node density is bounded by

$$
\lambda_{c}^{*} \leq \frac{\lambda_{c}}{\left(1+\sum_{i=0}^{k-2} p \times(i+2)^{-\alpha}\right)^{\frac{2}{\alpha}}} .
$$

where $\lambda_{c}$ is the critical node density for the non-cooperative network.

Proof: Refer to Fig. 1, for a given node $n_{0}$ in the cooperative network of infinite size, we find its nearest neighbor $n_{1}$ on the right side of $n_{0}$. Similarly, we find $n_{2}$ for $n_{1}$, $\cdots$, until we find $n_{k}$ for $n_{k-1}$. Nodes $n_{0} \sim n_{k-1}$ form a $k$-collaborative cluster to $n_{k}$. Given a positive $d_{c}$ defined by (4), $\forall i=0, \cdots, k-1$, we denote by $E_{i}$ the event that $d_{i, i+1} \leq d_{c}$ and $n_{i+1}$ is on the right side of $n_{i}$. We then have $\operatorname{Prob}\left\{E_{i}\right\}=\frac{1}{2} \operatorname{Prob}\left\{d_{i, i+1} \leq d_{c}\right\}$. Applying Lemma 2, we have

$$
\operatorname{Prob}\left\{E_{0} \bigcap E_{1} \cdots \bigcap E_{k-1}\right\}=\left(\frac{1}{2}\right)^{k}\left(1-e^{-\pi d_{c}{ }^{2} \lambda}\right)^{k}
$$

As mentioned earlier, (4) and $d_{c} \geq \max _{i=0, \cdots, k-1} d_{i, i+1}$ together ensure that the $k$-collaborative cluster (of any topology) with $p$-Selfishness transmits to $n_{k}$. If $n_{k+1}$ is within a distance $d_{c}$ away from $n_{k}$, we will have $d_{c} \geq \max _{i=1, \cdots, k} d_{i, i+1}$. Obviously, $n_{1}, n_{2}, \cdots, n_{k-1}$ and $n_{k}$ will further form a new $k$ collaborative cluster that can transmit to $n_{k+1}$. This procedure continues and an infinite cluster chain containing the given node $n_{0}$ appears almost surely. With (7), we know that the infinite cluster chain appears with non-zero probability. This indeed makes the network percolative by Definition 1 .

Let $r^{*}$ be the effective range of the cluster. Since $d_{c}$ is obtained for the worst-case cooperation and represent the effective range of the linear topology cluster, we have $d_{c} \leq r^{*}$. Let $\lambda_{c}^{*}$ be the critical node density for the $k$-collaborative network to percolate. By using the scale property (Lemma 1), we have

$$
\lambda_{c}^{*}=\lambda_{c} \times\left(r / r^{*}\right)^{2} \leq \lambda_{c} \times\left(r / d_{c}\right)^{2} .
$$

where $\lambda_{c}$ is the critical node density for the non-cooperative network to percolate.

Substituting (4) into (8) completes the proof.

\section{NUMERICAL RESULTS}

We numerically evaluate the critical density for a $k$ collaborative network to percolate. In the experiment, the critical density for the non-cooperative network is normalized to be 1. We plot in Fig. 3 the upper bound of the critical density for various $k$ and path loss exponent $\alpha$.

Fig. 3 verifies that node cooperation helps decrease system cost in terms of decreased node density, and when cooperation instead of selfishness dominates, i.e., cooperation probability $p>0.5$, the number of nodes needed in a cooperative network will be less than $77 \%$ of what is needed in a non-cooperative network, for the configuration: $k=20, \alpha=2.0$.

\section{COnClusions}

We analyzed the connectivity of $k$-collaborative network with selfishness. Unlike traditional work on cooperative networks, we do not assume that all nodes would like to transmit cooperatively when relaying other nodes' traffic. Specifically, we model nodes in such a way that each node cooperatively transmits with $p$-Selfishness. We then use stochastic geometry and percolation theory to study the connectivity of such system. Finally, we provide an upper bound of critical node density for the considered network.

\section{ACKNOWLEDGMENT}

This work was supported, in part, by Johns Hopkins University, Applied Physics Laboratory's internal research and development funds.

\section{REFERENCES}

[1] B. Liu, C. Westphal, D. Towsley, L. Wang, and D. Goeckel, "Asymptotic connectivity properties of cooperative wireless ad hoc networks," IEEE J. Sel. Areas Commun., vol. 27, no. 7, pp. 1226-1237, 2009.

[2] A. Bletsas, H. Shin, and M. Z. Win, "Outage analysis for co-operative communication with multiple amplify-and-forward relays," Electron. Lett., vol. 43, no. 6, pp. 51-52, 2007.

[3] H. Ding, J. Ge, and Z. Jiang, "Asymptotic performance analysis of amplify-and-forward with partial relay selection in Rician fading," Electron. Lett., vol. 46, no. 3, pp. 263-264, 2010.

[4] E. Liu, Q. Zhang, and K. K. Leung, "Residual energy-aware cooperative transmission (react) in wireless networks," in Proc. 19th Annual Wireless and Optical Communications Conf. (WOCC), 2010, pp. 1-6.

[5] J. Yang, A. G. Klein, and I. Brown, "Natural cooperation in wireless networks," IEEE Signal Process. Mag., vol. 26, no. 5, pp. 98-106, 2009.

[6] V. Srinivasan, P. Nuggehalli, C.-F. Chiasserini, and R. R. Rao, "An analytical approach to the study of cooperation in wireless ad hoc networks," IEEE Trans. Wireless Commun., vol. 4, no. 2, pp. 722-733, 2005.

[7] L. Wang, B. Liu, D. Goeckel, D. Towsley, and C. Westphal, "Connectivity in cooperative wireless ad hoc networks," in Proc. 9th ACM International Symposium on Mobile Ad Hoc Networking and Computing, 2008, pp. 121-130.

[8] R. Meester and R. Roy, Continuum Percolation. Cambridge University Press, 2008.

[9] H. T. Friis, "A note on a simple transmission formula," Proc. IRE, vol. 34, no. 5, pp. 254-256, 1946.

[10] F. Baccelli, O. Dousse, M. Haenggi, J. G. Andrews, and M. Franceschetti, "Stochastic geometry and random graphs for the analysis and design of wireless networks," IEEE J. Sel. Areas Commun., vol. 27, no. 7, pp. 1029-1046, 2009.

[11] D. Stoyan, W. S. Kendall, and J. Mecke, Stochastic Geometry and its Applications, 2nd edition. John Wiley \& Sons, 1996. 